\title{
A Look-Ahead Method for Computing Vector Padé-Hermite Approximants
}

\author{
Marc Van Barel and Adhemar Bultheel
}

\begin{abstract}
In this paper, we present an algorithm to compute vector Padé-Hermite approximants along a sequence of perfect points in the vector Padé-Hermite table. We show the connection to matrix Pade approximants. The algorithm is used to compute the solution of a block Hankel system of linear equations.
\end{abstract}

\section{Introduction}

Given a matrix of power series, we shall describe an algorithm for recursively computing the vector Pade-Hermite approximants that are located at successive perfect points on a diagonal of the vector Padé-Hermite table. In fact, our algorithm even computes a basis for all possible solutions that can be obtained in that point of the table.

A vector Padé-Hermite approximant with order indices $\boldsymbol{\sigma}$ for a matrix of power series $F(z) \in \mathbf{F}[[z]]^{s \times m}$ is a polynomial vector $\mathbf{p}(z) \in \mathbf{F}[z]^{m}$ such that

$$
F(z) \mathbf{p}(z)=O_{+}\left(z^{\sigma}\right) \text {. }
$$

When $s=1, m=2$, and $F(z)=[-1 f(z)]$, with $f(z)$ a scalar power series, we get a Padé approximant [1],[2]. When $s=1$, and $\sigma$, which reduces to a scalar integer number, is the sum of the allowed degrees of p plus $(m-1)$, then $\mathbf{p}$ is a Padé-Hermite approximant. For more details, we refer the interested reader to the introduction of [21],[8] and the references therein.

The vector Padé-Hermite table considered in this paper, is a generalization of the PadeHermite table [18],[14] and the classical Padé table [12]. The algorithms of [10],[19] are based on relationships between neighboring approximants in the Padé-Hermite table. However, the algorithms are only guaranteed to work when the vector of power series is perfect (see [14]). To overcome this restriction, we developed in [21] añ algorithm computing nonperfect Padé-Hermite approximants.

This algorithm is very similar to the one developed by Beckermann in [4] to solve the M-Padé approximation problem. When all the interpolation points coincide, the

Date received: January 27, 1994. Date revised: October 20, 1994. Communicated by Edward B. Saff. AMS classification: $41 \mathrm{~A} 21,65 \mathrm{~F} 05$.

Key words and phrases: (matrix) Padé approximation, (vector) Padé-Hermite approximation, (block) Hankel systems of equations, look-ahead, perfect points. 
M-Padé approximation problem reduces to a Padé-Hermite approximation problem. Beckermann also investigated the singular structure of the M-Padé table in [3]. In [20] the simultaneous Padé approximation problem was solved in a similar way. The previous two types of approximation and the more general matrix rational interpolation problems are all special cases of the vector M-Padé approximation problem. In [22], we derived basic algorithmic steps to follow any path of approximation. Beckermann and Labahn [5] introduced the concept of power Hermite-Padé approximants to solve different types of matrix Padé approximation problems. They even derived a superfast algorithm to accomplish this. The basic steps taken in all these algorithms are the most elementary steps one can take; i.e., adding only one new interpolation condition, changing only one degree of the interpolant. However, when using floating point arithmetic, this way of working does not (yet) allow control of the numerical stability of the algorithm.

It turns out that algorithms which make bigger jumps, but require a special structure of the solution, have more flexibility in controlling the stability. Originally such algorithms were not designed to overcome numerical stability problems but to overcome the nonperfectness of the given power series. The special structure of the solution is found in so-called perfect points. Hence, instead of doing elementary steps to go from one perfect point to another using some intermediate nonperfect points, these algorithms "look-ahead" from a perfect point immediately to the next perfect one.

The algorithm developed in this paper is such a "look-ahead" procedure. It can be seen as a first step towards a numerically (forward) stable algorithm when floating point arithmetic is used. Instead of jumping from one perfect point to the next one, the updating formulas derived here are also valid when going from one perfect point to any other later one. Hence, we could compute successive "well-conditioned" perfect points. For a successful adaptation of these ideas to the scalar Padé approximation problem, we refer to [9], and for the (scalar) Padé-Hermite approximation problem, to [6]. However, in this paper, we will not concentrate on these stability considerations. Another reason to have updating formulas for bigger jumps, not necessarily with a special structure of the solution, is the construction of superfast algorithms. See, for example, [5],[7],[13].

Our algorithm generalizes an algorithm of Cabay, Labahn and Beckermann [8], which treats a similar problem for the scalar Padé-Hermite case. Instead of taking a row vector of scalar power series, we replace each scalar power series by a column vector of power series. Hence, we get in general an $(s \times m)$ matrix of power series (with $s<m$ ). Cabay and Labahn also give a generalization in [7], the difference being that each scalar power series is replaced by a square matrix power series. Therefore, the number of columns of the resulting matrix of power series is a multiple of the number of rows. Hence, the problem they consider can be directly transformed into the problem solved here, but not vice-versa. However, they use the recurrence relation from one perfect point to $a$ later one to construct a superfast algorithm.

Besides generalizing the previous work of Cabay, Labahn and Beckermann, we wanted to develop the updating formulas that go from one perfect point to another as a special case of a very general and simple updating formula (allowing construction of superfast algorithms where the intermediate points don't need to be perfect). In spite of the simple general updating formula, it turns out that when special structure conditions are imposed, this formula becomes more detailed and complex. 
In Section 7, we also relate the problem to matrix Padé approximation and give the connection with [17]. In Section 8, we use the algorithm to compute the solution of block Hankel systems. We make the comparison with [15] where the inverse of a block Hankel matrix is computed.

Only minor modifications are needed to obtain an updating of the basis when moving from one perfect point to another that need not be on the same diagonal. Also, adaptations for a superfast version of the algorithms are standard and can be applied easily to our algorithm.

We start with some preliminary definitions and notations. Let $\mathbf{N}=\{0,1,2, \ldots\}$ be the set of natural numbers and $\mathbf{Z}$, the set of integers. An index $s$-tuple will be written in vector notation: e.g., $\sigma=\left(\sigma_{1}, \ldots, \sigma_{s}\right) \in \mathbf{N}^{s}$.

$\mathbf{F}$ is an arbitrary (finite or infinite) field. $\mathbf{F}[z]^{s \times m}$ and $\mathbf{F}[[z]]^{s \times m}$ denote the set of $s \times m$ polynomials and formal Maclaurin series, respectively. All formal Maclaurin series in this paper will be in $\mathbf{F}[[z]]^{s \times m}$ with $s<m$.

For $F(z) \in \mathbf{F}[[z]]^{s \times m}$ and $\boldsymbol{\sigma} \in \mathbf{N}^{s}$, we write

$$
F(z)=O_{+}\left(z^{\sigma}\right)
$$

to mean that the $(i, j)$-th entry of $F(z)$ is of the form

$$
f_{i j}(z)=\sum_{k \geq \sigma_{i}} f_{i j k} z^{k}
$$

i.e., $\sigma_{i}$ indicates the order of row $i$ of $F(z)$. We use an analogous definition for $O_{-}\left(z^{\sigma}\right)$.

For $\sigma \in \mathbf{Z}^{s}$ and $F(z) \in \mathbf{F}[[z]]^{s \times m}$, we write, $z^{\sigma} F(z)$, for $\operatorname{diag}\left(z^{\sigma_{1}}, \ldots, z^{\sigma_{s}}\right) F(z)$.

Let $\tau \in \mathbf{Z}^{m}$ and $\mathbf{p}(z) \in \mathbf{F}[z]^{m}$, then

$$
\tau-\operatorname{deg}\left(p_{1}(z), \ldots, p_{m}(z)\right)=\max \left\{\operatorname{deg} p_{i}(z)-\tau_{i}, i=1,2, \ldots, m\right\} .
$$

With such a polynomial vector $\mathbf{p}(z)$ of $\tau$-degree $\delta$ we associate its coefficient vector

$$
\operatorname{coeff}(\mathbf{p})=\left[p_{0,1}, p_{1,1}, \ldots, p_{\delta+\tau_{1}, 1}|\ldots| p_{0, m}, p_{1, m}, \ldots, p_{\delta+\tau_{m}, m}\right]^{T}
$$

where

$$
p_{i}(z)=\sum_{k=0}^{\delta+\tau_{i}} p_{k, i} z^{k}, \quad i=1,2, \ldots, m
$$

For a general polynomial matrix

$$
P(z)=\left[\mathbf{p}_{1}(z), r_{2}(z), \ldots, \mathbf{p}_{k}(z)\right],
$$

we say that $\tau-\operatorname{deg} P(z)=\alpha \in \mathbf{Z}^{k}$ when $\tau-\operatorname{deg} \mathbf{p}_{i}(z)=\alpha_{i}$ for $i=1, \ldots, k$. If each of the columns is considered to have a $\tau$-degree $\leq \delta$, we define the coefficient matrix of $P(z)$ as

$$
\operatorname{coeff}(P)=\left[\operatorname{coeff}\left(\mathbf{p}_{1}\right), \ldots, \operatorname{coeff}\left(\mathbf{p}_{k}\right)\right],
$$

with $\operatorname{coeff}\left(\mathbf{p}_{j}\right), j=1,2, \ldots, k$, given by (1).

For more details about the following concepts, we refer to [22].

If $\mathbf{p}(z) \in \mathbf{F}[z]^{m}$ and $\tau$-deg $\mathbf{p}(z)=\delta$, then $\tau$-hdc $\mathbf{p}(z)$ is the highest degree coefficient with respect to $\tau$, that is, the vector in $\mathbf{F}^{m}$ whose $i$-th component equals the coefficient 
of $z^{\delta+\tau_{i}}$ in $p_{i}(z)$. A set of polynomial vectors is $\tau$-reduced iff their $\tau$-hdc vectors are linearly independent.

For an $s$-tuple $\mathbf{z}=\left(z_{1}, \ldots, z_{s}\right) \in \mathbf{Z}^{s}$,

$$
\|\mathbf{z}\|=\sum_{i=1}^{s}\left|z_{i}\right| .
$$

We define a function vect $: \mathbf{N} \rightarrow \mathbf{N}^{s}: t \mapsto \operatorname{vect}(t)=\sigma=\left(\sigma_{1}, \sigma_{2}, \ldots, \sigma_{s}\right)$ by

$$
\sigma_{1}=\sigma_{2}=\cdots=\sigma_{r}=q+1 \text { and } \sigma_{r+1}=\cdots=\sigma_{s}=q
$$

where $q$ and $r$ are defined by the unique decomposition of $t$ into $t=q s+r$, with $0 \leq r<s$ (i.e., $r=t \bmod s$ ). Note that $\|\sigma\|=\|\operatorname{vect}(t)\|=t$, so that $\sigma$ is uniquely defined by its norm.

The rationale behind this definition is that if we have $t$ interpolation conditions, to be distributed over an $s$ dimensional vectorial interpolation problem and $\sigma=\operatorname{vect}(t)$, then a natural choice is to distribute these conditions cyclically such that $\sigma_{i}$ conditions are imposed on component $i$. Such ordering was proposed for the first time by Van Iseghem [23].

In this paper, we shall assume that the vectors $\sigma$ of order indices (sec below) are always in this natural ordering, so that $\sigma=\operatorname{vect}(\|\sigma\|)$ and hence that $\sigma$ is uniquely defined by its norm.

We also reserve the notation $\mathbf{e}_{s}=(1, \ldots, 1,0, \ldots, 0) \in Z^{m}$ with $s$ ones and $\mathbf{e}=$ $(1, \ldots, 1) \in \mathbf{Z}^{m}$. By $\varepsilon$ we mean the $s$-dimensional analogue of $\mathbf{e}$.

\section{Vector Padé-Hermite Approximation}

We are interested in the approximants, defined as follows.

Definition 1 (vector Padé-Hermite approximation). Given $F(z) \in \mathbf{F}[[z]]^{s \times m}$ with $s<$ $m$, we say that a polynomial vector $\mathbf{p}(z) \in \mathbf{F}[z]^{m \times 1}$ is a vector Padé-Hermite approximant with order indices $\sigma \in \mathbf{N}^{s}$ for $F(z)$ if it satisfies the vector interpolation conditions

$$
F(z) \mathbf{p}(z)=O_{+}\left(z^{\sigma}\right) .
$$

$S^{\sigma}$ denotes the set of all vector Padé-Hermite approximants for given $\sigma$ and given $F(z)$.

Recall that the vectors $\sigma$ are supposed to be in the natural ordering, so that $\sigma$, and hence also $S^{\sigma}$, is completely fixed by the natural number $t=\|\sigma\|$.

We define the vector Padé-Hermite table as follows.

Definition 2 (vector Padé-Hermite table). Let the formal $s \times m$ Maclaurin series $F(z) \in$ $\mathbf{F}[[z]]^{s \times m}$ with $s<m$ be given. Point $\tau \in \mathbf{N}^{m}$ of the $m$-dimensional vector Padé-Hermite table of $F(z)$ contains a matrix $M_{\tau}(z)=\left[\mathbf{b}_{1}(z), \mathbf{b}_{2}(z), \ldots, \mathbf{b}_{\alpha}(z)\right]$ whose columns form a basis of the $\mathbf{F}$-vector space $S_{\tau} . S_{\tau}$ is the set of all polynomial vectors $\mathbf{p}(z)$ that have $\tau$-degree $\leq 0$ and are vector Padé-Hermite approximants for $F(z)$ with order indices $\boldsymbol{\sigma}=\operatorname{vect}(\|\boldsymbol{\tau}\|+s)$. 
Note that the vector of degree indices, $\tau$, gives an upper bound for the degrees that we allow for the polynomial components of $\mathbf{p}(z)$, but at the same time fixes the number of interpolation conditions by $\|\sigma\|=\|\tau\|+s$. Hence, it determines $\sigma$ and therefore the specific interpolation conditions that are to be satisfied. In other words, $S_{\tau} \subset S^{\sigma}$ contains all the elements $\mathbf{p}(z) \in S^{\sigma}$ with $\tau$-deg $\mathbf{p}(z) \leq 0$. The converse is not true; $\sigma$ will not define $\tau$ uniquely. The previous connection between the degree and order indices, i.e., $\boldsymbol{\sigma}=\operatorname{vect}(\|\boldsymbol{\tau}\|+s)$, will be maintained in the rest of the paper.

The computation of vector Padé-Hermite approximants is equivalent to the solution of a structured system of homogeneous linear equations. This can be seen as follows. We denote the columns of $F(z)$ by $\mathbf{f}_{j}(z)$, where

$$
\mathbf{f}_{j}(z)=\sum_{k=0}^{\infty} \mathbf{f}_{k, j} z^{k} \quad \text { with } \quad \mathbf{f}_{k, j} \in \mathbf{F}^{s \times]} \quad\left(\mathbf{f}_{k, j}=\mathbf{0} \text { for } k<0\right) .
$$

The elements of $S_{\tau}$ can be found by computing the solutions of the following set of linear homogeneous equations, which incorporates the degree conditions as well as the corresponding interpolation conditions,

$$
T_{\tau} \operatorname{coeff}(\mathbf{p})=0 .
$$

The generalized block Sylvester matrix $T_{\tau}$ is defined as follows $(t=\|\sigma\|=\|\tau\|+s)$ :

$$
T_{\tau}=\left[T_{\tau, 1}\left|T_{\tau, 2}\right| \ldots \mid T_{\tau, m}\right] \in \mathbf{F}^{t \times(\|\tau\|+m)},
$$

where

with

$$
T_{\tau, j}=\left[\begin{array}{cccc}
\mathbf{f}_{0, j} & & & \\
\mathbf{f}_{1, j} & \mathbf{f}_{0, j} & & \\
\vdots & \vdots & \ddots & \\
\mathbf{f}_{q-1, j} & \mathbf{f}_{q-2, j} & \cdots & \mathbf{f}_{q-\tau_{j}-1, j} \\
\mathbf{f}_{q, j}^{*} & \mathbf{f}_{q-1, j}^{*} & \cdots & \mathbf{f}_{q-\tau_{j}, j}^{*}
\end{array}\right] \in \mathbf{F}^{t \times\left(\tau_{j}+1\right)}
$$

$$
\mathbf{f}_{i, j}^{*} \in \mathbf{F}^{r \times l}
$$

containing the first $r$ elements of $\mathbf{f}_{i, j}$ with $r=\|\boldsymbol{\tau}\| \bmod s$ (recall $t=\|\boldsymbol{\sigma}\|=\|\tau\|+s=$ $s q+r)$. The number of unknowns is $\|\tau+\mathbf{e}\|=\|\tau\|+m$. The number of equations (interpolation conditions) is $\|\sigma\|=\|\tau\|+s$. Because $s<m$, the basis $M_{\tau}(z)$ will at least contain $(\|\boldsymbol{\tau}\|+m)-(\|\boldsymbol{\tau}\|+s)=m-s$ elements.

Definition 3 (perfect point). If the basis for $S_{\tau}$ contains precisely $m-s$ elements, we say that $\tau$ is a perfect point.

The following theorems are simple observations.

Theorem 4 (characterization of a perfect point). The point $\tau$ is perfect iff $T_{\tau}$ has full rank, i.e. iff the $\|\tau\|+s$ rows of $T_{\tau}$ are linearly independent.

Note that the non-trivial part of the first block row of $T_{\tau}$ is equal to the constant coefficient of $F(z)$, i.e., $F(0)$. This implies the following theorem. 
Theorem 5. Let $T_{\tau}^{*}$ be equal to $T_{\tau}$ but with the first block row and the first column of each of the $m$ block columns deleted. The generalized block Sylvester matrix $T_{\tau}$ has full rank iff $F(0)$ has full rank and the square generalized block Sylvester matrix $T_{\tau}^{*}$ is nonsingular.

In the sequel we assume that $F(0)$ has full rank and that the columns of $F(z)$ are ordered such that the first $s$ columns of $F(0)$ are linearly independent.

Corollary 6. Assume that $F(0)$ has full rank. Then the following are equivalent.

1. The point $\tau$ is perfect.

2. $T_{T}$ has full rank.

3. $T_{T}^{*} \in \mathbf{F}^{\|\boldsymbol{\tau}\| \times\|\boldsymbol{\tau}\|}$ is nonsingular.

4. There is no nontrivial solution with $\tau$-degree $\leq-1$ satisfying the first $\|\tau\|$ interpolation conditions.

The following theorem says that for a perfect point, a basis matrix for $S_{\tau}$ will always be $\tau$-reduced.

Theorem 7 ( $\boldsymbol{\tau}$-reduced basis matrix). Let $\boldsymbol{\tau}$ be a perfect point for $F(z) \in \mathbf{F}[[z]]^{s \times m}$ and let

$$
M_{\tau}(z)=\left[\mathbf{b}_{1}(z), \mathbf{b}_{2}(z), \ldots, \mathbf{b}_{\alpha}(z)\right]
$$

be a basis matrix for $S_{\tau}$. Then $\alpha=m-s$ and $M_{\tau}(z)$ is $\tau$-reduced with $\tau$-degree 0 .

Proof. Suppose $M_{\tau}(z)$ is not $\tau$-reduced, i.e., the $\tau$-highest degree coefficient of one of the basis vectors is already zero or can be made equal to zero by recombination of the basis vectors. However, multiplying this basis vector by $z$ would give a nontrivial solution satisfying (3) with the constant term equal to zero. Hence, there would exist a nontrivial solution of

$$
T_{\tau}^{*} \operatorname{coeff}(\mathbf{p})=0
$$

This means that $T_{\tau}^{*}$ is singular, hence $\tau$ is not a perfect point which contradicts our assumption.

Theorem 8. If $\boldsymbol{\tau}$ is a perfect point for $F(z) \in \mathbf{F}[[z]]^{s \times m}$ and $M_{\tau}(z)$ is a basis matrix for $S_{\tau}$, then the $m-s$ columns of $M_{\tau}(0)$ are linearly independent. Moreover, if the columns $i_{1}, i_{2}, \ldots, i_{s}$ of $F(0)$ are linearly independent (form a nonsingular matrix), then the $(m-s) \times(m-s)$ matrix obtained by deleting rows $i_{1}, i_{2}, \ldots, i_{s}$ in $M_{\tau}(0)$ is nonsingular.

Proof. If the basis vectors have linearly dependent constant coefficients, they can be combined to give a nontrivial solution of (3) which has the constant term equal to zero. As in the previous proof, this means that $\tau$ can not be a perfect point. To prove the second part, observe that $F(0) M_{\tau}(0)=0$ is the first block row of (3). 


\section{Basis Matrix for $S^{\sigma}$}

In this section we look for a compact way to denote all elements of $S^{\sigma}$, i.e., all the solutions of (2). For more details and proofs, we refer to [22].

Because $S^{\sigma}$ forms an $\mathbf{F}[z]$-submodule of $\mathbf{F}[z]^{m}$, we can represent $\mathbf{p}(z) \in S^{\sigma}$ as a polynomial linear combination of some basis vectors $\mathbf{b}_{1}(z), \ldots, \mathbf{b}_{m}(z)$. Arranging these basis vectors as the columns of a basis matrix $B^{\sigma}(z) \in \mathbf{F}[z]^{m \times m}$, we see that all solutions $\mathbf{p}(z)$ of $(2)$ can be written as

$$
\mathbf{p}(z)=B^{\sigma}(z) \mathbf{c}(z),
$$

with $\mathbf{c}(z) \in \mathbf{F}^{m \times 1}[z]$. This $\mathbf{e}(z)$ contains the uniquely defined coordinates of $\mathbf{p}(z)$ with respect to this basis matrix.

To construct and identify a basis matrix $B^{\sigma}(z)$ we can use the following characterization theorem.

Theorem 9 (characterization of a basis matrix). From [22]. A polynomial matrix $B(z) \in \mathbf{F}^{m \times m}[z]$ is a basis matrix for $S^{\sigma}$ iff

1. the $m$ columns $\mathbf{b}_{j}(z)$ of $B(z)$ belong to $S^{\sigma}$, i.e., they satisfy (2);

2. $\operatorname{deg} \operatorname{det} B(z)=k_{S}$ with $k_{S}$ the number of independent conditions out of the $\|\sigma\|$ interpolation conditions given by (2).

Remarks. 1. $k_{S}$ is minimal in the following sense. Take $m$ polynomial vectors $\mathbf{p}_{j}(z)$, $j=1,2, \ldots, m$ satisfying (2) and set

$$
P(z)=\left[\mathbf{p}_{1}(z), \mathbf{p}_{2}(z), \ldots, \mathbf{p}_{m}(z)\right] .
$$

If $\operatorname{det} P(z)$ is not identically 0 , then $\operatorname{deg} \operatorname{det} P(z) \geq k_{S}$.

2. If $F(0)$ has full rank $s$, all interpolation conditions are linearly independent, i.e., $k_{S}=\|\boldsymbol{\sigma}\|$.

All elements of $S^{\sigma}$ of limited $\tau$-degree can be represented as follows.

Theorem 10 ( $\tau$-reduced basis matrix). From [22]. If the basis matrix

$$
B^{\sigma}(z)=\left[\mathbf{b}_{1}(z), \mathbf{b}_{2}(z), \ldots, \mathbf{b}_{m}(z)\right]
$$

for $S^{\sigma}$ is $\tau$-reduced, then all the elements of $S^{\sigma}$ having $\tau$-degree $\leq \alpha$ can be represented as

$$
\mathbf{p}(z)=B^{\sigma}(z) \mathbf{c}(z),
$$

with $\operatorname{deg} c_{i}(z) \leq \alpha-\alpha_{i}$ and $\alpha_{i}=\tau$-deg of $\mathbf{b}_{i}(z)$.

The latter result will allow us to construct an entry $M_{\tau}(z)$ of the vector Padé-Hermite table corresponding to a perfect point $\tau$. By the previous theorem it is obvious that for a perfect point $\tau$, we can generate the subset $S_{\tau}$ of all elements in $S^{\sigma}$ with $\tau$-degree $\leq 0$, where $\sigma=\operatorname{vect}(\|\tau\|+s)$ if we have a $\tau$-reduced basis matrix $B^{\sigma}(z)$ for the submodule $S^{\sigma}$. Thus the construction of a basis matrix $M_{\tau}(z)$ for the vector space $S_{\tau}$ is reduced to the construction of a $\tau$-reduced basis matrix $B_{\tau}(z)$ for the submodule $S^{\sigma}$.

We use the following definition. 
Definition 11 (residual series, residual). If $\mathbf{p}(z) \in S^{\sigma}$, we define the residual series $R(z) \in \mathbf{F}[[z]]^{s \times 1}$ of $\mathbf{p}(z)$ with respect to $F(z)$ for the order indices $\sigma$ as

$$
F(z) \mathbf{p}(z)=z^{\sigma} R(z) \text {. }
$$

The constant coefficient $R(0)$ of $R(z)$ is called the residual of $\mathbf{p}(z)$.

Theorem 12. Let $\tau$ be a perfect point of $F(z) \in \mathbf{F}[[z]]^{s \times m}$ and $M_{\tau}(z)$ a basis matrix which is the entry of the Padé-Hermite table at point $\tau$. The matrix

$$
B_{\tau}(z)=[\underbrace{z^{2} B_{\tau}^{*}(z)}_{s} \underbrace{M_{\tau}(z)}_{m-s}]
$$

with

$$
T_{\tau}^{*} \operatorname{coeff}\left(B_{\tau}^{*}\right)=\left[0_{s \times(\|\tau\|-s)}, X\right]^{T}, \quad X \in \mathbf{F}^{s \times s} \text { nonsingular, }
$$

and $\tau-\operatorname{deg} B_{\tau}^{*}(z) \leq-\mathbf{e}$ (columnwise), forms a $\tau$-reduced basis matrix for $S^{\sigma}$ with $\boldsymbol{\sigma}=$ vect $(\|\tau\|+s)$. The $\tau$-column degrees of $B_{\tau}(z)$ are $\mathbf{e}_{s}$ (s ones, $m-s$ zeros). Hence, $\operatorname{deg} \operatorname{det} B_{\tau}(z)=\|\sigma\|$. In other words, the columns of $B_{\tau}^{*}(z)$ are vector Padé-Hermite approximants for order indices $\sigma-2 \varepsilon$, having $\tau$-degree $=-1$ and having linearly independent residuals.

Proof. First, note that $B_{\tau}^{*}(z)$ can always be computed because $T_{\tau}^{*}$ is nonsingular if $\tau$ is a perfect point. Second, note that all the columns of $B_{\tau}$ are in $S^{\sigma}$.

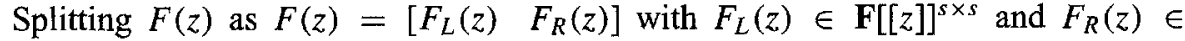
$\mathbf{F}[[z]]^{s \times(m-s)}$, it holds that

$$
\left[\begin{array}{cc}
F_{L}(z) & F_{R}(z) \\
O_{(m-s) \times s} & I_{m-s}
\end{array}\right] B_{\tau}(z)=\left[\begin{array}{cc}
z^{\sigma} X+O_{+}\left(z^{\sigma+\varepsilon}\right) & \times \\
0_{(m-s) \times s} & M_{\tau}^{\prime}(0)+O_{+}\left(z^{1}\right)
\end{array}\right],
$$

with $M_{\tau}^{\prime}(z)$ the last $m-s$ rows of $M_{\tau}(z)$. Taking the determinant of the left and right hand side, we derive that

$$
0 \neq \operatorname{det} B_{\tau}(z)=O_{+}\left(z^{\|\sigma\|}\right) .
$$

On the other hand, the $\tau$-deg of the columns of $M_{\tau}(z)$ are 0 and the $\tau$-deg of the columns of $B_{\tau}^{*}$ are $\leq-1$.

Therefore,

$$
z^{-\tau} B_{\tau}(z)=B_{h} z^{\mathbf{e}_{s}}+O_{-}\left(z^{\mathbf{e}_{s}-\mathbf{e}}\right)
$$

or

$$
\operatorname{det} B_{\tau}(z)=\operatorname{det} B_{h} z^{\|\tau\|+s}+O_{-}\left(z^{\|\tau\|+s-1}\right)=O_{-}\left(z^{\|\sigma\|}\right) .
$$

Hence, we get that det $B_{\tau}(z)=\operatorname{det} B_{h} z^{\|\sigma\|} \neq 0$. Thus, $\operatorname{deg} \operatorname{det} B_{\tau}(z)=\|\sigma\|=k_{S}$, and by Theorem 9 , this $B_{\tau}$ is a basis matrix for $S^{\sigma}$. Moreover, because the highest degree coefficients of the columns of $B_{\tau}(z)$ are the corresponding columns of $B_{h}$ and det $B_{h} \neq 0, B_{\tau}(z)$ is $\tau$-reduced with $\tau$-column degrees $\mathbf{e}_{s}$.

In the sequel, we need a uniquely defined basis matrix in a perfect point.

Theorem 13. Let $\boldsymbol{\tau}$ be a perfect point of $F(z) \in \mathbf{F}[[z]]^{s \times m}$. Then, there exists a unique $\tau$-reduced basis matrix $B_{\tau}(z)$ for $S^{\sigma}$ such that 
1. it has the form $B_{\tau}(z)=\left[z^{2} B_{L}(z) B_{R}(z)\right]$ with $B_{L}(z) \in \mathbf{F}[z]^{m \times s}$ and $B_{R}(z) \in$ $\mathbf{F}[z]^{m \times(m-s)}$;

2. the $\tau$-column degrees of $B_{\tau}(z)$ are $\mathbf{e}_{s}$;

3. $F(z) z^{2} B_{L}(z)=z^{\sigma} I_{s}+O_{+}\left(z^{\sigma+\varepsilon}\right)$;

4. $B_{R}^{\prime}(0)=I_{m-s}$ where $B_{R}^{\prime}(z)$ denotes the last $m-s$ rows of $B_{R}(z)$.

Proof. The left part, $B_{L}(z)$, is uniquely determined by the second and third conditions or

$$
T_{\tau}^{*} \operatorname{coeff}\left(B_{L}\right)=\left[0_{s \times(\|\tau\|-s)}, I_{s}\right]^{T} .
$$

Hence, $B_{L}(z)=B_{\tau}^{*}$ with $X=I_{s}$ in the previous theorem. The right part, $B_{R}(z)$, is uniquely determined by the second and fourth conditions. We get that $B_{R}(z)=M_{\tau}(z)$ with the last $m-s$ rows of $M_{\tau}(0)$ equal to $I_{m-s}$. From the previous theorem, we see that the matrix $\left[B_{L}(z) B_{R}(z)\right]$ is a $\tau$-reduced basis matrix for $S^{\sigma}$.

Definition (canonical basis matrix). Let $\boldsymbol{\tau}$ be a perfect point of $F(z) \in \mathbf{F}[[z]]^{s \times m}$. The unique basis matrix from the previous theorem is called the canonical basis matrix.

\section{Going from One Perfect Point to Another One}

Assume that we know a $\tau$-reduced basis matrix $B_{\tau}(z)$ for $S^{\sigma}$ corresponding to the perfect point $\tau$ given by Theorem 12 . Suppose that $\tau+k \mathbf{e}$ is also a perfect point. Can we compute a $(\tau+k \mathbf{e})$-reduced basis matrix $B_{\tau+k \mathbf{e}}(z)$ starting from the basis matrix $B_{\tau}(z)$ ? The following observation is trivial.

Lemma 15 ( $\tau$-reduced). A polynomial matrix $P(z) \in \mathbf{F}[z]^{m \times m}$ is $\tau$-reduced iff $P(z)$ is $(\tau+k \mathbf{e})$-reduced. Moreover,

$$
\tau-\operatorname{deg}(P)=(\tau+k \mathbf{e})-\operatorname{deg}(P)+k \mathbf{e} .
$$

When adding interpolation conditions, we can use a general updating theorem. It says that a basis with order indices $\sigma+\nu$ can be obtained by combining an order $\sigma$ basis for $F(z)$ with an order $\nu$ basis for its residual series. In the sequel, we will need basis matrices of submodules based on other series than the series $F(z)$. From now on, if it is not clear which series is used, we will include it in the notation as follows: $B^{\sigma}(F ; z)$ and $S^{\sigma}(F)$.

Theorem 16 (general updating theorem). Let $B^{\sigma}(F ; z)$ be a $\tau$-reduced basis matrix for $S^{\sigma}(F)$. The $\tau$-degrees of the columns of $B^{\sigma}(F ; z)$ are $\alpha$. The polynomial matrix $B^{\sigma+\nu}(F ; z)=B^{\sigma}(F ; z) B^{\nu}(R ; z)$ is a $\tau$-reduced basis matrix for $S^{\sigma+\nu}(F)$ iff $B^{\nu}(R ; z)$ is a $(-\alpha)$-reduced basis matrix for the submodule $S^{\nu}(R)$, with

$$
S^{\nu}(R)=\left\{\mathbf{p}(z) \mid R(z) \mathbf{p}(z)=O_{+}\left(z^{\nu}\right)\right\},
$$

where $R(z)$ is the residual series of $B^{\sigma}(F ; z)$, i.e., $F(z) B^{\sigma}(F ; z)=z^{\sigma} R(z)$.

Proof. A proof of this theorem can be found in Appendix A. 
We have to be careful when we apply this theorem to update from one perfect point to another one. Suppose $\|\boldsymbol{\sigma}+\nu\|=q^{\prime} s+r^{\prime}, 0 \leq r^{\prime}<s$. Then we impose $q^{\prime}+1$ interpolation conditions for the rows $1, \ldots, r^{\prime}$ of $F(z)$ and $q^{\prime}$ interpolation conditions for the rows $r^{\prime}+1, \ldots, s$. If $\|\sigma\|=q s+r, 0 \leq r<s$, then the elements from $S^{\sigma}(F)$ satisfy $q+1$ interpolation conditions for the rows $1, \ldots, r$ and $q$ conditions for the rows $r+1, \ldots, s$. So the order $\nu$ approximant for the residual should exactly fill up the difference in conditions per row. Thus the interpolation conditions for the residuals are imposed on the $\|\boldsymbol{\nu}\|$ rows, repeated cyclically, starting with row $r+1$. In other words, the order $\nu$ approximant for the residual series $R(z)$ is in fact computed for $C^{r} R(z)$, where $C$ represents the permutation operator which shuffles the rows cyclically one place up. Applying the previous theorem to $B_{\tau}(F ; z)$ and $B_{\tau+k \mathbf{e}}(F ; z)$, results in the following theorem.

Theorem 17 (updating from one perfect point to another). Let $\tau$ and $\tau+k \mathbf{e}$ be two perfect points on a diagonal of the Padé-Hermite table for $F(z)$. Suppose $B_{\tau}(F ; z)$ is a $\tau$-reduced basis for $S^{\sigma}(F)$, with $\sigma=$ vect $(\|\tau\|+s)$ (e.g., as given by Theorem 12). Let $R_{\tau}(z)$ be the residual series of $B_{\tau}(F ; z)$, i.e., $F(z) B_{\tau}(F ; z)=z^{\sigma} R_{\tau}(z)$. Finally, for $0<k \in \mathbf{N}$, define $\nu \in \mathbf{N}^{s}$ as the vector of order indices $\nu=\operatorname{vect}(\mathrm{km})$.

Then the polynomial matrix $B_{\tau+k \mathrm{e}}(F ; z)=B_{\tau}(F ; z) B_{k \mathbf{e}-\mathrm{e}_{s}}\left(C^{r} R_{\tau} ; z\right)$ is $a(\tau+k \mathbf{e})$ reduced basis matrix for $S^{\sigma+\nu}\left(C^{r} R_{\tau}\right)$ iff $B_{k \mathbf{e}-\mathbf{e}_{s}}\left(C^{r} R_{\tau} ; z\right)$ is a $\left(k \mathbf{e}-\mathbf{e}_{s}\right)$-reduced basis matrix for $S^{\nu}\left(C^{r} R_{\tau}\right)$ for the perfect point $\left(k \mathbf{e}-\mathbf{e}_{s}\right)$ in the vector Padé-Hermite table for $C^{r} R_{\tau}(z)$ with $\|\sigma\|=q s+r, 0 \leq r<s$.

Proof. Let us first prove that if $\tau$ is a perfect point for $F(z)$, then $\tau+k e$ is also a perfect point for $F(z)$ iff $\left(k \mathbf{e}-\mathbf{e}_{s}\right)$ is a perfect point for $R_{\tau}^{c}(z)=C^{r} R_{\tau}(z)$. First assume that $\left(k \mathrm{e}-\mathbf{e}_{s}\right.$ ) is not a perfect point for $R_{\tau}^{c}(z)$. Hence, there exists a non-trivial polynomial vector $\mathbf{p}(z)$ having $\left(k \mathbf{e}-\mathbf{e}_{s}\right.$ )-degree $\leq-1$ satisfying the first $\left\|k \mathbf{e}-\mathbf{e}_{s}\right\|=\mathrm{km}-\mathrm{s}$ interpolation conditions.

Note that $\mathbf{q}(z)=B_{\tau}(F ; z) \mathbf{p}(z)$ has $(\tau+k \mathbf{e})$-degree $\leq-1$ and $\mathbf{q}(z)$ is a nontrivial solution satisfying the first

$$
(\|\boldsymbol{\tau}\|+s)+\left\|k \mathbf{e}-\mathbf{e}_{s}\right\|=\|\boldsymbol{\tau}+k \mathbf{e}\|
$$

interpolation conditions for $F(z)$. Hence, $\tau+k e$ is not a perfect point.

For the "if"-part, we assume that $\left(k \mathbf{e}-\mathbf{e}_{s}\right)$ is a perfect point for $R_{\tau}^{c}(z)$ and, as above, it follows easily that $\tau+k \mathbf{e}$ is a perfect point for $F(z)$.

The rest of the theorem follows immediately from Lemma 15 and Theorem 16.

Note that if $B_{\tau}(F ; z)$ and $B_{k \mathbf{e}-\mathbf{e}_{s}}\left(C^{r} R_{\tau} ; z\right)$ are constructed using Theorem 12 , also $B_{\tau}(F ; z) B_{k e-e_{s}}\left(C^{r} R_{\tau} ; z\right)$ will have the form described in this theorem.

\section{The Border of the Vector Padé-Hermite Table}

In the previous section, we have assumed that $\tau \geq \mathbf{0}$ (componentwise). Theorem 17 shows how to jump from the perfect point $\tau$ to the perfect point $\tau+k \mathbf{e}$.

Suppose now that we are interested in a $\tau$-reduced basis matrix $B_{\tau}(z)$ for the submodule $S^{\sigma}$ where some of the components of $\tau$ may be negative. The first nontrivial point 
on the diagonal $\tau+k \mathbf{e}, k \in \mathbf{Z}$ is

$$
\tau-\tau_{\max } \mathbf{e}, \quad \text { with } \tau_{\max }=\max \left\{\tau_{j} \mid j=1,2, \ldots, m\right\} .
$$

However, some of the components of $\tau-k \mathbf{e}, k=\tau_{\max }, \tau_{\max }-1, \ldots, 0$ can be negative. Hence, we have to extend the definition of $S^{\sigma}, B_{\tau}(z), S_{\tau}, M_{\tau}(z), \ldots$ whenever some $\tau_{j}<0$.

Definition 18 (projection of the diagonal $\tau-k e$ ). We define the projection of $\mathbf{z}=$ $\left(z_{1}, \ldots, z_{m}\right) \in \mathbf{Z}^{m}$ on the positive cone by $\operatorname{proj}(\mathbf{z})=\pi$ with

$$
\pi_{j}=\max \left\{0, z_{j}\right\}, \quad j=1, \ldots, m .
$$

We call the piecewise linear path $\pi_{k}=\operatorname{proj}\left(\tau_{k}\right)$, the projection of the diagonal path $\tau_{k}=\tau-k \mathrm{e}, k=\tau_{\max }, \tau_{\max }-1, \ldots, 0$, when each point is replaced by its projection on the positive cone. When $\exists \tau_{j}: \tau<0$, the entities $B_{\tau}(z), S_{\tau}, M_{\tau}(z), \ldots$ are defined as the corresponding entities for the projection $\pi=\operatorname{proj}(\tau)$. Thus, for example, $B_{\tau}(z)=$ $B_{\pi}(z)$.

We now show that the results of the previous section are also valid when $\exists \tau_{j}: \tau_{j}<0$. From now on, we shall assume, without loss of generality, that the columns of $F(z) \in$ $\mathbf{F}[[z]]^{s \times m}$ are ordered such that for a perfect point $\tau, \sum_{j=1}^{s} \tau_{j}$ is maximal with $F_{L}(0)$ nonsingular. We use $F_{L}(z)$ to denote the $s \times s$ matrix containing the first $s$ columns of $F(z)$. Note that this special ordering will only be necessary to establish the results when $\tau \neq \operatorname{proj}(\tau)$, i.e., on the border of the vector Padé-Hermite table.

Theorem 19. Given $\tau \in \mathbf{Z}^{m}$ and $F(z) \in \mathbf{F}[[z]]^{s \times m}$. The columns of $F(z)$ are ordered as explained above.

Let the point $\pi=\operatorname{proj}(\tau)$ be perfect. Let $B_{\tau}(z)=B_{\pi}(z)$ be the canonical $\pi$-reduced basis matrix given by Theorem 12. Then, $B_{\tau}(z)$ is also a $\tau$-reduced basis matrix for $S^{\sigma}, \sigma=$ vect $(\|\pi\|+s)$. Its $\pi$-column degrees are $\mathbf{e}_{s}$ and its $\tau$-column degrees are $\mathbf{e}_{s}+(\boldsymbol{\pi}-\boldsymbol{\tau})$. Moreover, the first s components of $(\boldsymbol{\pi}-\boldsymbol{\tau})$ are always zero.

Proof. The proof of this theorem is given in Appendix B.

We now extend Theorem 17 to the more general case when one of the components of $\tau$ could be negative.

Theorem 20 (updating from one perfect point to another). Let $B_{\tau}(F ; z)=B_{\pi}(F ; z)$, with $\pi=\operatorname{proj}(\tau)$, the canonical $\tau$-reduced basis matrix for $S^{\sigma}(F), \sigma=\operatorname{vect}(\|\pi\|+s)$, given by Theorem 19.

For $0<k \in \mathbf{N}$, the polynomial matrix $B_{\tau}(F ; z) B_{\delta}\left(R_{\tau}^{c} ; z\right)$ is the canonical $(\tau+k \mathbf{e})$ reduced basis matrix $B_{\tau+k \mathbf{e}}^{-}(F ; z)=B_{\pi^{\prime}}(F ; z)$ for $S^{\sigma+\nu}(F)$, with $\pi^{\prime}=\operatorname{proj}(\tau+k \mathbf{e})$ for the perfect point $\tau+k \mathbf{e}$ iff $B^{\nu}\left(R_{\tau}^{c} ; z\right)=B_{\delta}\left(R_{\tau}^{c} ; z\right)$ is the canonical $\delta=k \mathbf{e}-\mathbf{e}_{s}-(\pi-\tau)$ reduced basis matrix for the perfect point $\delta$ in the vector Padé-Hermite table for $R_{\tau}^{c}(z)=$ $C^{r} R_{\tau}(z)$, with $R_{\tau}(z)$ the residual series for $B_{\tau}(z)$ (i.e. $F(z) B_{\tau}(F ; z)=z^{\sigma} R_{\tau}(z), C$ is the cyclical up-shift matrix, and $r=\|\sigma\| \bmod s$ ).

The $\delta$-column degrees of $B_{\delta}\left(R_{\tau}^{c} ; z\right)$ are $\mathbf{e}_{s}+\left(\boldsymbol{\pi}^{\prime}-\tau-k \mathbf{e}\right)$. 
Proof. The proof of this theorem is given in Appendix C.

\section{Algorithm}

The previous theorem allows us to construct an algorithm jumping from one perfect point to the next, on the diagonal $\tau-l \mathbf{e}, l=\tau_{\max }, \tau_{\max }-1, \ldots$ in the vector Padé-Hermite table.

\section{Algorithm 21 Diagonal Path.}

Input: $F(z) \in \mathbf{F}[[z]]^{s \times m}$, with $F(0)$ of full rank and $\tau \in \mathbf{Z}^{m}$.

Step $l$ (a) permute the columns of $F(z)$ and the corresponding elements of $\tau$ such that $F_{L}(0)$ is nonsingular with $\sum_{j=1}^{s} \tau_{j}$ maximal;

(b) $k \leftarrow 1 ; \mu \leftarrow \tau-\left(\tau_{\max }+1\right) \mathbf{e} ; \pi \leftarrow-\mathbf{e}_{s} ; B_{\mu}(F ; z) \leftarrow I_{m} ; R_{\mu}^{c}(z) \leftarrow F(z)$.

Step 2 for $l=\tau_{\max }, \tau_{\max }-1, \ldots, 0$

(a) $\delta \leftarrow k \mathbf{e}-\mathbf{e}_{s}-(\pi-\mu)$

(b) $d \leftarrow \operatorname{det}\left(T_{\delta}^{*}\right)$ with $T_{\delta}^{*}$ based on $R_{\mu}^{c}(z)$

(c) if $d \neq 0$ then

(1) construct the canonical $B_{\delta}\left(R_{\mu}^{c} ; z\right)$ from Theorem 12

(2) $B_{\mu+k \mathrm{e}}(F ; z) \leftarrow B_{\mu}(F ; z) B_{\delta}\left(R_{\mu}^{c} ; z\right)$

(3) $R_{j b+k \mathrm{e}}^{\prime}(z) \leftarrow z^{-\nu} R_{\mu}^{c}(z) B_{\delta}(z)$ with $\nu=\operatorname{vect}(\|\delta\|+s)$

(4) $\mu \leftarrow \mu+k \mathbf{e}$

(5) $\pi \leftarrow \operatorname{proj}(\mu)$

(6) $R_{\mu}^{c}(z)=C^{r} R_{\mu}^{\prime}(z)$ with $r=\|\delta\| \bmod s$

(7) $k \leftarrow 1$

else

(1) $k \leftarrow k+1$

endif

endfor

Output: if $\mu=\tau$ then

(a) $\tau$ is a perfect point;

(b) $B_{\mu}(F ; z)$ is a $\mu$-reduced basis matrix connected to this perfect point $\mu$. else

(a) $\tau$ is not a perfect point;

(b) $\mu$ is the last perfect point on the diagonal before $\tau$ and $B_{\mu}(F ; z)$ is the corresponding basis matrix;

(c) if we are interested in a $\tau$-reduced basis matrix $B_{\tau}(F ; z)$ for the non-perfect point $\tau$, we can compute a $\delta$-reduced basis matrix $B_{\delta}\left(R_{\mu}^{c} ; z\right)$ for the nonperfect point $\delta$ for $R_{\mu}^{c}(z)$. The polynomial matrix $B_{\mu}(F ; z) B_{\delta}\left(R_{\mu}^{c} ; z\right)$ is a $\tau$-reduced basis matrix for the non-perfect point $\tau$.

\section{endif}

Remarks. 1. When $\mu$ is initialized as $\mu \leftarrow \tau-\left(\tau_{\max }+1\right) \mathbf{e}$ and $\pi$ as $\pi \leftarrow-\mathbf{e}_{s}$, we start by looking for a first perfect point of the form

$$
(\delta \leftarrow) k \mathbf{e}-\mathbf{e}_{s}-(\boldsymbol{\pi}-\mu)=\tau-\left(\tau_{\max }+1\right) \mathbf{e}+k \mathbf{e}
$$

with $k>0$. 
2. In a manner that is analogous to [8], we can show that this algorithm requires only $O\left(\|\operatorname{proj}(\tau)\|^{2}\right)$ FLOPS if the variable $k$ only reaches small values compared to $\|\operatorname{proj}(\tau)\|$.

3. Suppose $s=1$ and $m=2$, with $F(z)=[-1 f(z)]$ (Padé-approximation) with $f(z)=O_{+}\left(z^{1}\right)$. We are interested in a $\tau$-reduced basis matrix $B_{\tau}(z)$ with $\tau=\left(\tau_{1}, \tau_{2}\right)$ and $0 \leq \tau_{1}<\tau_{2}$. Hence, $\tau_{2}=\tau_{\max }=\max \left\{\tau_{1}, \tau_{2}\right\}$. If we follow the diagonal path $\tau-l \mathbf{e}, l=\tau_{2}, \tau_{2}-1, \ldots, \tau_{1}, \ldots, 0$, the first point which is possibly a perfect point, is reached for $l=\tau_{1}$. Indeed, we have shown that the first $s$ components of $\pi=\operatorname{proj}(\tau)$ are equal to the corresponding components of $\tau$ for a perfect point $\tau$. However,

$$
\tau_{1}-l \neq \pi_{1}=0, \quad l=\tau_{2}, \tau_{2}-1, \ldots \tau_{1}+1 .
$$

Also $l=\tau_{1}$ gives no perfect point. This also follows from the fact that the first row of $T_{\pi}^{*}$ is a zero row with $\pi=\operatorname{proj}(\tau-l \mathbf{e}), l=\tau_{2}, \tau_{2}-1, \ldots, \tau_{1}$.

Suppose $l=\tau_{1}-1$ gives the first perfect point $\tau$. Computing a $\tau$-reduced basis matrix $B_{\tau}(z)$ for this perfect point, requires $O\left(\left(\tau_{2}-\tau_{1}\right)^{3}\right)$ FLOPS. Hence, if $\tau_{2} \gg \tau_{1}$, then the variable $k$ of Algorithm 21 reaches the value $\tau_{2}-\tau_{1}+1$, which is not moderate anymore compared to the total number of interpolation conditions.

Therefore, to reduce the amount of computational work, one could follow other paths in the vector Padé-Hermite table. These paths are chosen such that the distance between two consecutive perfect points is small compared to the total length of the path. Of course, when a non-diagonal step is made, the updating formulas have to be adapted.

4. Algorithm 21 jumps from one perfect point to the next one on a diagonal path in the vector Padé-Hermite table. The updating formula of Theorem 20 , however, is valid for stepping from one perfect point to any that follow it. Hence, instead of choosing the next perfect point, we can choose one of the "well-conditioned" perfect points that follow it. That is, instead of determining the next point based on the determinant of $T_{\delta}^{*}$, we can look for a $\delta$ such that the condition number of $T_{\delta}^{*}$ is small enough, which implies that this also determines a perfect point.

5. Instead of Algorithm 21, a divide and conquer strategy could be used as in Cabay and Labahn [7], reducing the amount of computational work to $O\left(k \log _{2}^{2} k\right)$, with $k=\|\operatorname{proj}(\tau)\|$.

\section{Matrix Padé Approximation}

In this section and the next one, we give two applications of the previous algorithm to related problems such as matrix Padé approximation, and the solution of block Hankel systems. Both problems can be reduced to a vector Padé-Hermite problem.

We get the following connection between the vector Padé-Hermite approximation problem and matrix Padé approximation.

We split $F(z) \in \mathbf{F}[[z]]^{s \times m}$ as

$$
F(z)=\left[F_{L}(z) F_{R}(z)\right]
$$

with

$$
F_{L}(z) \in \mathbf{F}[[z]]^{s \times s} \text { and } F_{R}(z) \in \mathbf{F}[[z]]^{s \times(m-s)},
$$

and assume that $F_{L}(0)$ is nonsingular. 
Let $\tau$ be a perfect point, and the basis matrix for the $F$-vector space $S_{\tau}$ be

$$
M_{\tau}(z)=\left[\mathbf{b}_{1}(z), \mathbf{b}_{2}(z), \ldots, \mathbf{b}_{m-s}(z)\right]
$$

This basis matrix can be split up as

$$
M_{\tau}(z)=\left[\begin{array}{c}
N(z) \\
D(z)
\end{array}\right], \quad \text { with } N(z) \in \mathbf{F}[z]^{s \times(m-s)} \text { and } D(z) \in \mathbf{F}[z]^{(m-s) \times(m-s)} .
$$

Because $F_{l}(0)$ is nonsingular, $D(0)$ is nonsingular (see Theorem 8 ). Hence, by rewriting the interpolation condition (2), we obtain

$$
F_{L}(z) N(z) D(z)^{-1}=-F_{R}(z)+O_{+}\left(z^{\sigma}\right)
$$

As usual $\sigma=\operatorname{vect}(\|\pi\|+s)$. If all the entries of $\sigma$ are the same, we get

$$
N(z) D(z)^{-1}=-F_{L}(z)^{-1} F_{R}(z)+O_{+}\left(z^{\sigma}\right) .
$$

If $F_{L}(z)=I_{s \times s}$, then, even if the entries of $\sigma$ are different from each other, we can always write

$$
N(z) D(z)^{-1}=-F_{R}(z)+O_{+}\left(z^{\sigma}\right)
$$

Hence, the $M_{\tau}(z)$ for a perfect point $\tau$ gives us a matrix Padé approximant having $\tau$-McMillan degree 0 . For the definition of $\tau$-McMillan degree, we refer to [22].

If $m=2 s$ and $\operatorname{proj}(\tau)=\tau=\left(\delta_{N}, \ldots, \delta_{N}, \delta_{D}, \ldots, \delta_{D}\right)\left(s\right.$ times $\delta_{N}$ and $\left.\delta_{D}\right)$, we get that $\sigma=\left(\delta_{N}+\delta_{D}+1\right) \varepsilon$. Hence, $N(z) D(z)^{-1}$ is a right matrix Padé fraction as defined in [17] with $\operatorname{deg} N(z) \leq \delta_{N}$ and $\operatorname{deg} D(z) \leq \delta_{D}$. When we use our algorithm for this specific data and jump immediately to the diagonal part of the path, we get a similar algorithm as in [17].

\section{Solution of Block Hankel Systems of Equations}

Let us consider the $(\alpha \times \beta)$ block Hankel matrix

$$
H=\left[\begin{array}{cccc}
H_{0} & H_{1} & \cdots & H_{\beta} \\
H_{1} & H_{2} & \cdots & H_{\beta+1} \\
\vdots & \vdots & \ddots & \vdots \\
H_{\alpha} & H_{\alpha+1} & \cdots & H_{\alpha+\beta}
\end{array}\right] \text {, with } H_{i} \in \mathbf{F}^{s \times q}
$$

Consider the set of linear equations

$$
H \mathbf{x}=\mathbf{b}, \quad \text { with } \quad \mathbf{b}=\left[\mathbf{b}_{0}^{T}, \mathbf{b}_{1}^{T}, \ldots, \mathbf{b}_{\alpha}^{T}\right]^{T} \quad \text { and } \quad \mathbf{b}_{i} \in \mathbf{F}^{s \times 1},
$$

where we look for

$$
\mathbf{x}=\left[\mathbf{x}_{\beta}^{T}, \mathbf{x}_{\beta-1}^{T}, \ldots, \mathbf{x}_{0}^{T}\right]^{T}, \quad \text { with } \quad \mathbf{x}_{i} \in \mathbf{F}^{q \times 1} .
$$

Before we can apply the previous techniques, we have to translate this linear algebra problem into a vector Padé-Hermite approximation problem. Let us rewrite the original 
linear algebra problem in the following equivalent one:

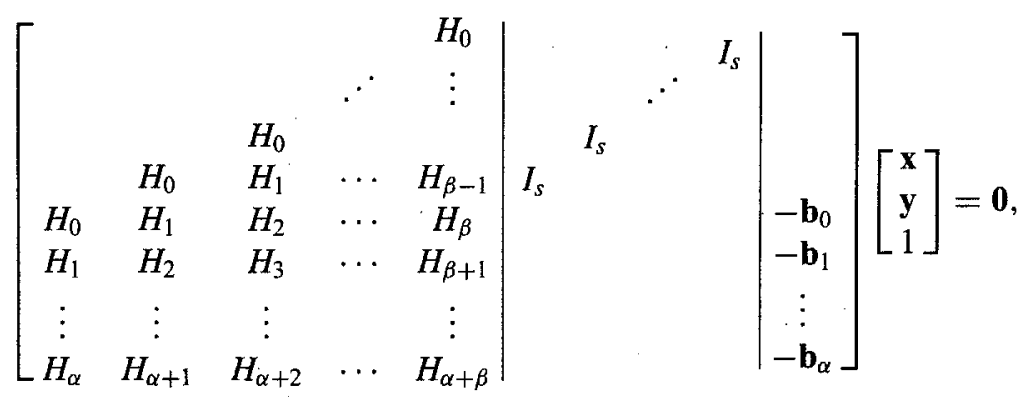

with unknowns $\mathbf{x}$ and $\mathbf{y}=\left[\mathbf{y}_{\beta-1}^{T}, \mathbf{y}_{\beta-2}^{T}, \ldots, \mathbf{y}_{0}^{T}\right]^{T}$ with $\mathbf{y}_{i} \in \mathbf{F}^{s \times 1}$. Note that this set of linear homogeneous equations has $(\alpha+\beta+1) s$ equations and $(\beta+1) q+\beta s+1$ unknowns (counting the 1 as an unknown).

If we define

$$
\begin{aligned}
H(z) & =H_{0} z^{0}+H_{1} z^{1}+\cdots+H_{\alpha+\beta} z^{\alpha+\beta}+O_{+}\left(z^{\alpha+\beta+1}\right), \\
\mathbf{b}(z) & =\mathbf{b}_{0} z^{0}+\mathbf{b}_{1} z^{1}+\cdots+\mathbf{b}_{\alpha} z^{\alpha}+O_{+}\left(z^{\alpha+1}\right), \\
\mathbf{x}(z) & =\mathbf{x}_{0} z^{0}+\mathbf{x}_{1} z^{1}+\cdots+\mathbf{x}_{\beta} z^{\beta}, \\
\mathbf{y}(z) & =\mathbf{y}_{0} z^{0}+\mathbf{y}_{1} z^{1}+\cdots+\mathbf{y}_{\beta-1} z^{\beta-1}
\end{aligned}
$$

equation (4) is equivalent to the following vector Padé-Hermite approximation problem:

$$
\left[\begin{array}{lll}
H(z) & I_{s} & \left.-z^{\beta} \mathbf{b}(z)\right]
\end{array}\right]\left[\begin{array}{c}
\mathbf{x}(z) \\
\mathbf{y}(z) \\
1
\end{array}\right]=O_{+}\left(z^{\alpha+\beta+1}\right),
$$

with $\mathbf{0}-\operatorname{deg} \mathbf{x} \leq \beta$ and $\mathbf{0}-\operatorname{deg} \mathbf{y} \leq \beta-1$. This can be rewritten as

$$
F(z) \mathbf{p}(z)=O_{+}\left(z^{\sigma}\right),
$$

with

$$
\begin{aligned}
m & =q+s+1, \\
F(z) & =\left[\begin{array}{lll}
H(z) I_{s} & \left.-z^{\beta} \mathbf{b}(z)\right] \in \mathbf{F}[[z]
\end{array}\right]^{s \times m}, \\
\mathbf{p}(z) & =\left[\begin{array}{lll}
\mathbf{x}^{T}(z) \mathbf{y}^{T}(z) & 1
\end{array}\right]^{T} \in \mathbf{F}[z]^{m \times 1}, \\
\boldsymbol{\tau}-\operatorname{deg} \mathbf{p} & \leq 0, \\
\boldsymbol{\tau} & =(\underbrace{\beta, \beta, \ldots, \beta}_{q}, \underbrace{\beta-1, \beta-1, \ldots, \beta-1}_{s}, 0), \\
\sigma & =(\alpha+\beta+1, \alpha+\beta+1, \ldots, \alpha+\beta+1) .
\end{aligned}
$$

Because $F(0)$ has full rank, we can use Algorithm 21 to compute $\tau$-reduced basis matrices $B_{\mu}(z)$ for each perfect point on the diagonal $\tau-l \mathbf{e}, l=\beta, \beta-1, \beta-2, \ldots, l^{*}$. Note that it might be that the columns of $F(z)$ should be reordered such that $F_{L}(0)$ is nonsingular with $\sum_{j=1}^{s} \tau_{j}$ maximal. The last value of $l$ (i.e., $l^{*}$ ) is chosen such that the number of interpolation conditions that determine $B_{\tau}(z)$ is smaller than or equal to the 
total number of given interpolation conditions, or

$$
l^{*}=\min \{l \in \mathbf{Z} \mid\|\boldsymbol{\pi}\|+s \leq(\alpha+\beta+1) s \quad \text { with } \pi=\operatorname{proj}(\boldsymbol{\tau}-l \mathbf{e})\} .
$$

Equivalently, $l^{*}$ is determined such that

$$
l^{*}=\min \{\{l \in \mathbf{Z} \mid(s+q) l \geq q \beta-(\alpha+1) s\} \cup\{\beta\}\} .
$$

From now on let us assume that the block Hankel matrix $H$ is square, i.e., $s(\alpha+1)=$ $q(\beta+1)$. Moreover, let $H$ be nonsingular. Hence, the solution space of $(4)$ has dimension 1 and there is only one element from this solution space having last component equal to 1.

Equation (5) simplifies to $l^{*}=0$. For $l=l^{*}$, we have that $\mu=\tau$ and the corresponding number of interpolation conditions is $\|\tau\|+s=(s+q) \beta$. The remaining number of interpolation conditions is

$$
(\alpha+\beta+1) s-(\|\tau\|+s)=q>0 .
$$

Hence, to obtain the final solution, we look for the unique solution of

$$
C^{r} R_{\tau}(z) \mathbf{p}(z)=O_{+}\left(z^{\nu}\right), \quad r=\|\tau\| \bmod s
$$

with

$$
\begin{aligned}
F(z) B_{\tau}(z) & =z^{\sigma^{\prime}} R_{\tau}(z), \\
\boldsymbol{\sigma}^{\prime} & =\operatorname{vect}(\|\boldsymbol{\tau}\|+s), \\
\nu & =\operatorname{vect}(q), \\
\left(-\mathbf{e}_{s}\right)-\operatorname{deg} \mathbf{p}(z) & \leq 0,
\end{aligned}
$$

and the last component of $\mathbf{p}(z)$ is equal to 1 .

Equation (6) translates into a set of $q$ linear homogeneous equations with $q+1$ unknowns. The solution space has dimension one and there exists a unique solution $\mathbf{p}(z)$ with the last component equal to one. The final solution $\mathbf{x}(z)$ of the block Hankel system of equations is given as

$$
\mathbf{x}(z)=\left[\begin{array}{lll}
I_{q} & 0 & 0
\end{array}\right] B_{\tau}(z) \mathbf{p}(z) .
$$

Suppose now that the last perfect point for $l=\beta, \beta-1, \ldots, 0$ is not $\tau-l^{*} \mathbf{e}=\tau$ but a previous point $\boldsymbol{\tau}-l \mathbf{e}$ with $l>0$. Now, $\mathbf{p}(z)$ is the unique solution of

$$
C^{r} R_{\tau-l \mathbf{e}}(z) \mathbf{p}(z)=O_{+}\left(z^{\nu}\right), \quad r=\|\operatorname{proj}(\tau-l \mathbf{e})\| \bmod s,
$$

with

$$
\begin{aligned}
F(z) B_{\tau-l \mathbf{e}}(z) & =z^{\sigma^{\prime}} R_{\tau-l \mathbf{e}}(z), \\
\sigma^{\prime} & =\operatorname{vect}(\|\operatorname{proj}(\tau-l \mathbf{e})\|+s), \\
\nu & =\operatorname{vect}\left(s(\alpha+\beta+1)-\left\|\sigma^{\prime}\right\|\right),
\end{aligned}
$$

i.e., $\|\nu\|=q(l+1)+s l,\left(l \mathbf{e}-\mathbf{e}_{s}-l \mathbf{u}_{s+q+1}\right)-\operatorname{deg} \mathbf{p}(z) \leq 0$ (for $l<\beta$ ), and the last component of $\mathbf{p}(z)$ is equal to one $\left(u_{i}=(0,0, \ldots, 1, \ldots, 0)\right.$ with the one at position $i$ ). Translating (8) into a linear algebra problem results in a set of $q(l+1)+s l$ linear homogeneous equations with $q(l+1)+s l+1$ unknowns. Scaling the last component 
to be one, gives us the unique $\mathbf{p}(z)$ which, together with (7), leads to the final solution of the block Hankel problem. In the case where the block Hankel matrix $H$ is singular, the solution space of $(6)$ and (8) will have dimension greater than one. From the basis vectors with the last component scaled to be one, all solutions of the block Hankel problem can be expressed using (7).

We could also solve the block Hankel system by using the inversion formula of Gohberg and Shalom [11]. The entities needed in this inversion formula can be immediately extracted from the $\tau$-reduced basis matrix for $S^{\sigma}$, given $F(z)$ with

$$
\begin{aligned}
& F(z)=\left[H_{-1}+z H(z)-I_{s}\right], \quad \text { with } H_{-1} \text { and } H_{\alpha+\beta+1} \text { arbitrary, } \\
& \boldsymbol{\tau}=(\beta+1) \mathbf{e}, \\
& \sigma=(\alpha+\beta+3) \varepsilon \text {. }
\end{aligned}
$$

Hence, we can use our algorithm to compute this basis matrix, going from one perfect point to the next. Note that the last point will be perfect if the block Hankel matrix is nonsingular. In [15], Labahn also gives an algorithm jumping from one perfect point to another. However, he only takes into account the perfect points $\tau$ such that $\sigma=\operatorname{vect}(\|\tau\|+s)$ has equal components. This means that the number of interpolation conditions that are added in each step is at least equal to the common multiple of $s$ and $q$ multiplied by $(1+s / q)$, while our algorithm adds at least $s+q$ interpolation conditions. Take, for example, $s=5$ and $q=7$. The method of Labahn uses a jump size of at least $35(1+3 / 5)=56$, while our algorithm uses a jump size of at least 12 . Hence, if there are intermediate perfect points between the perfect points found by the algorithm of Labahn, our algorithm will be more efficient. When the blocks are square, this difference disappears. We refer the interested reader to [16] where the inversion components of block Hankel-like matrices are computed.

\section{Conclusion}

In this paper, we have constructed an algorithm going from one perfect point to another on a diagonal path in a vector Padé-Hermite table. We have indicated that a slightly modified version of the algorithm can also be used to compute basis matrices for "wellconditioned" perfect points or to compute the solution of a block Hankel system of equations.

\section{References}

1. G. A. BAKer, JR. AND P. R. Graves-Morris (1981): Padé Approximants. Part I: Basic Theory, volume 13 of Encyclopedia Math. Appl., Addison-Wesley, Reading, MA.

2. G. A. BAKER, JR. AND P. R. Graves-Morris (1981); Padé Approximants. Part II: Extensions and Applications, volume 14 of Encyclopedia Math. Appl., Addison-Wesley, Reading, MA.

3. B. BECKERMANN (1990): The structure of the singular solution table of the M-Pade approximation problem. J. Comput. Appl. Math., 32(1\&2):3-15.

4. B. BeCKermann (1992): A reliable method for computing M-Padé approximants on arbitrary staircases. J. Comput. Appl. Math., 40:19-42.

5. B. BECKERMANN AND G. LABAHN (1992): A uniform approach for Hermite-Padé and simultaneous Padé approximants and their matrix-type generalizations. Numer. Alg., 3(1-4):45-54. 
6. S. CABAY, A. R. Jones, AND G. LABAHN (1994): A stable algorithm for multi-dimensional Padé systems and the inversion of generalized Sylvester matrices. Technical Report TR 94-07, Department of Computing Science, University of Alberta, Edmonton, Alberta, Canada.

7. S. CABAY AND G. LABAHN (1992): A superfast algorithm for multi-dimensional Padé systems. Numerical Algorithms, 2:201-224.

8. S. CABAY, G. Labahn, AND B. BeckermanN (1992): On the theory and computation of nonperfect Padé-Hermite approximants. J. Comput. Appl. Math., 39:295-313.

9. S. CABAY AND R. MeleshKo (1993): A weakly stable algorithm for Padé approximants and the inversion of Hankel matrices. SIAM J. Matrix Anal. Appl., 14:735-765.

10. J. Della Dora And C. Di Crescenzo (1984): Approximants de Padé-Hermite. Numer. Math., 43:23-57.

11. I. C. GoHBERG AND T. SHALOM (1990): On Bezoutians of nonsquare matrix polynomials and inversion of matrices with nonsquare blocks. Lin. Alg. Appl., 249-323.

12. W. B. GRAGG (1972): The Padé table and its relation to certain algorithms of numerical analysis. SIAM Rev, 14:1-62.

13. M. H. GUTKNECHT (1993): Stable row recurrences for the Pade table and generically superfast lookahead solvers for non-Hermitian Toeplitz systems. Lin. Alg. Appl., 188/189:351-422.

14. H. JAGer (1964): A multidimensional generalization of the Padé table. Proc. Kon. Nederl. Akad. Wet., 67:193-249. (Indagationes Mathematicae, 26).

15. G. LABAHN (1990): Inversion algorithm for rectangular-block Hankel matrices. Technical Report CS-9052, Univ. of Waterloo, Dept. Computer Sci.

16. G. LABAHN (1992): Inversion components of block Hankel-like matrices. Lin. Alg. Appl., 177:7-48.

17. G. LABAHN AND S. CABAY (1989): Matrix Padé fractions and their computation. SIAM J. of Computing, 18(4):639 657 .

18. K. MAHLER (1968): Perfect systems. Compositio Math., 19:95-166.

19. S. PAszKowski (1987): Recurrence relations in Padé-Hermite approximation. J. Comput. Appl. Math., 19(1):99-107.

20. M. VAN BAREL AND A. BultheEL (1990) A new approach to the rational interpolation problem: the vector case. J. Comput. Appl. Math., 33(3):331-346.

21. M. Van Barel and A. Bultheel (1991): The computation of non-perfect Padé-Hermite approximants. Numer. Alg., 1:285-304.

22. M. VAN BAREL AND A. Bultheel (1992): A general module theoretic framework for vector M-Padé and matrix rational interpolation. Numer. Alg., 3:451-461.

23. J. VAN IsEghem (1986): Vector Padé approximants. In R. Vichnevetsky and J. Vignes, editors, Numerical Mathematics and its Applications, 73-77, Amsterdam, North-Holland.

\section{Appendix A. Proof of Theorem 16}

When $F(0)$ has full rank, we have the following result for the constant coefficient of a basis matrix $B^{\sigma}(z)$.

Theorem 22. Given $F(z) \in \mathbf{F}[[z]]^{s \times m}$ with $s<m$. Take any basis matrix $B^{\sigma}(z)$ for the submodule $S^{\sigma}$ given by Definition 1. If the columns $i_{1}, i_{2}, \ldots, i_{s}$ of $F(0)$ are linearly independent, then the $(m-s) \times m$ matrix $D B^{\sigma}(0)$ obtained by deleting rows $i_{1}, i_{2}, \ldots, i_{s}$ in $B^{\sigma}(0)$ has full rank. The matrix $D$ is obtained by deleting rows $i_{1}, i_{2}, \ldots, i_{s}$ in the $m \times m$ identity matrix. Moreover,

$$
\left[\begin{array}{c}
R(0) \\
D B^{\sigma}(0)
\end{array}\right]
$$

is nonsingular where $R(z)=z^{-\sigma} F(z) B^{\sigma}(z)$, i.e., the residual series of $B^{\sigma}(z)$. Hence, $R(0)$ and $D B^{\sigma}(0)$ have full rank. Also, $\operatorname{deg} \operatorname{det} B^{\sigma}(z)=\|\sigma\|$, i.e., all interpolation conditions are linearly independent. 
Proof. Consider the following equality:

$$
\left[\begin{array}{c}
F(z) \\
D
\end{array}\right] B^{\sigma}(z)=\left[\begin{array}{c}
z^{\sigma} R(z) \\
D B^{\sigma}(z)
\end{array}\right]
$$

or

$$
B^{\sigma}(z)=\left[\begin{array}{c}
F(z) \\
D
\end{array}\right]^{-1}\left[\begin{array}{c}
z^{\sigma} R(z) \\
D B^{\sigma}(z)
\end{array}\right]
$$

Because

$$
\begin{aligned}
\operatorname{det} B^{\sigma}(z) & =O_{-}\left(z^{\|\sigma\|}\right), \\
\operatorname{det}\left[\begin{array}{c}
F(z) \\
D
\end{array}\right]^{-1} & =O_{+}\left(z^{0}\right), \\
\operatorname{det}\left[\begin{array}{c}
z^{\sigma} R(z) \\
D B^{\sigma}(z)
\end{array}\right] & =O_{+}\left(z^{\|\sigma\|}\right),
\end{aligned}
$$

we see that $\operatorname{det} B^{\sigma}(z)=c z^{\|\sigma\|}$, with $0 \neq c \in \mathbf{F}$, and that

$$
\left[\begin{array}{c}
R(0) \\
D B(0)
\end{array}\right]
$$

is nonsingular. Hence, $D B(0)$ and $R(0)$ have full rank, i.e., the rank is, respectively, $m-s$ and $s$.

The proof of Theorem 16 can now be given.

Proof. The polynomial matrix $B^{\sigma}(z) \in \mathbf{F}[z]^{m \times m}$ is a $\tau$-reduced basis matrix for $S^{\sigma}$, given $F(z)$, iff

1. $F(z) B^{\sigma}(z)=z^{\sigma} R(z)$, with $R(z) \in \mathbf{F}[[z]]^{s \times m}$,

2. $\operatorname{deg} \operatorname{det} B^{\sigma}(z)=\|\sigma\|$,

3. $B^{\sigma}(z)=z^{\tau} B_{h}^{\sigma}(z) z^{\alpha}$, with $B_{h}^{\sigma}(z)=O_{-}\left(z^{0}\right)$ and deg det $B_{h}^{\sigma}(z)=0$.

$\Leftarrow$ The polynomial matrix $B^{\nu}(z)$ is a $(-\alpha)$-reduced basis matrix for $S^{\nu}$, given $R(z)$, iff

a. $R(z) B^{\nu}(z)=z^{\nu} R^{\prime}(z), \quad$ with $R^{\prime}(z) \in \mathbf{F}[[z]]^{s \times m}$,

b. $\operatorname{deg} \operatorname{det} B^{\nu}(z)=\|\nu\|$,

c. $B^{\nu}(z)=z^{-\alpha} B_{h}^{\nu}(z) z^{\beta}$, with $B_{h}^{\nu}(z)=O_{-}\left(z^{0}\right)$ and $\operatorname{deg} \operatorname{det} B_{h}^{\nu}(z)=0$.

Then the polynomial matrix $B^{\sigma+\nu}(z)=B^{\sigma}(z) B^{\nu}(z)$ is a $\tau$-reduced basis matrix for $S^{\sigma}$, given $F(z)$, because

a. $F(z) B^{\sigma+\nu}(z)=F(z)\left(B^{\sigma}(z) B^{\nu}(z)\right)=z^{\sigma} R(z) B^{\nu}(z)=z^{\sigma+\nu} R^{\prime}(z)$,

b. $\operatorname{deg} \operatorname{det} B^{\sigma}(z)=\|\sigma\|+\|\nu\|=\|\sigma+\nu\|$,

c. $B^{\sigma+\nu}(z)=\left(z^{\tau} B_{h}^{\sigma}(z) z^{\alpha}\right)\left(z^{-\alpha} B_{h}^{\nu}(z) z^{\beta}\right)=z^{\tau} B_{h}^{\sigma+\nu}(z) z^{\beta}$, with $B_{h}^{\sigma+\nu}(z)=$ $B_{h}^{\sigma}(z) B_{h}^{\nu}(z)=O_{-}\left(z^{0}\right)$ and $\operatorname{deg} \operatorname{det} B_{h}^{\sigma+\nu}(z)=0$.

$\Rightarrow$ In a similar way as for the "if" part, one can show that $B^{\nu}(z)=\left(B^{\sigma}(z)\right)^{-1} B^{\sigma+\nu}(z)$ satisfies all the conditions to be a $(-\alpha)$-reduced basis matrix for $S^{\nu}$, given $R(z)$, except that it is a polynomial matrix. With the notation of the previous theorem, we 
have the following equality

$$
\left[\begin{array}{c}
R(z) \\
D B^{\sigma}(z)
\end{array}\right] B^{\nu}(z)=\left[\begin{array}{c}
z^{\nu} R^{\prime}(z) \\
D B^{\sigma+\nu}(z)
\end{array}\right]
$$

or

$$
\begin{aligned}
B^{\nu}(z) & =\left[\begin{array}{c}
R(z) \\
D B^{\sigma}(z)
\end{array}\right]^{-3}\left[\begin{array}{c}
z^{\nu} R^{\prime}(z) \\
D B^{\sigma+\nu}(z)
\end{array}\right] \\
& =O_{+}\left(z^{0}\right) O_{+}\left(z^{0}\right) \\
& =O_{+}\left(z^{0}\right) .
\end{aligned}
$$

Hence, $B^{\nu}(z)$ is polynomial.

\section{Appendix B. Proof of Theorem 19}

Proof. Let us first prove the last statement in Theorem 19, i.e., that $\pi_{j}=\tau_{j}, j=$ $1,2, \ldots, s$. Suppose there is some $k, 1 \leq k \leq s$ such that $\pi_{k} \neq \tau_{k}$. Note that this is only possible when $0=\pi_{k}>\tau_{k}$. Hence, $B_{\tau}(0)$ has the following form:

$$
B_{\tau}(0)=\left[\begin{array}{cc|cccc}
0 \ldots 0 & * * * & * \ldots & \ldots \\
\hline 0_{(m-s) \times s} & & \multicolumn{1}{c}{I_{m-s}}
\end{array}\right] \longleftarrow k
$$

We now prove that all $*$-entries are zero. Suppose the $(k, j)$-entry, $0<k \leq s<j \leq m$, of $B_{r}(0)$ is different from zero. Because $F(0) B_{\tau}(0)=0$, we could switch columns $k$ and $j$ of $F(z)$, keeping $F_{L}(0)$ nonsingular. Hence, $\tau_{j} \leq \tau_{k}<0\left(\sum_{i=1}^{s} \tau_{i}\right.$ is maximal) or the $j$-th row of $B_{\tau}(z)$ contains only terms of degree 0 . Therefore, by expanding the determinant of $B_{\tau}(2)$ along row $j$, we see that it is equal to the determinant of a square matrix of size $m-1$, which has the same form as (9). Hence, by induction on the dimension of these matrices, one can prove that, if $0=\pi_{k}>\tau_{k}$ with $1 \leq k \leq s$, then det $B_{\tau}(z)=0$. This contradicts the fact that $B_{\tau}(z)$ is a basis matrix. Hence $\pi_{j}=\tau_{j}$, for all $j=1,2, \ldots, s$.

To prove the remainder of the theorem, we assume without loss of generality that $0=\pi_{j}>\tau_{j}, j=s+1, \ldots, s+k$, and $\pi_{j}=\tau_{j}$ for other $j$ values. Hence, the $\pi$-reduced basis matrix $B_{\tau}(z)$ can be written as

$$
B_{\tau}(z)=z^{\pi}\left[\begin{array}{c|cc}
* & * & * \\
\hline 0 & I_{k} & 0 \\
* & * & *
\end{array}\right] z^{\mathbf{e}_{s}}=z^{\pi} B_{\tau, h}(z) z^{\mathbf{e}_{s}},
$$

with deg det $B_{\tau, h}(z)=0$ and $B_{\tau, h}(z)=O_{-}\left(z^{0}\right)$ (componentwise). Therefore, $B_{\tau}(z)$ can also be written as a $\tau$-reduced basis matrix,

$$
\begin{aligned}
B_{\tau}(z) & =z^{\tau}\left[z^{\pi-\tau} B_{\tau, h}(z) z^{\tau-\pi}\right] z^{\pi-\tau+\mathbf{e}_{i}} \\
& =z^{\tau} B_{\tau, h}^{\prime}(z) z^{\mathbf{e}_{s}+\pi-\tau}
\end{aligned}
$$

where, from the structure of $B_{\tau, h}(z)$, it also follows that $\operatorname{deg} \operatorname{det} B_{r, h}^{\prime}(z)=0$ and $B_{\tau, h}^{\prime}(z)=O_{-}\left(z^{0}\right)$. This proves the theorem. 


\section{Appendix C. Proof of Theorem 20}

Proof. Similar to the first part of the proof of Theorem 17, we can show that $\tau+k \mathbf{e}$ is a perfect point for $F(z)$ iff $\delta=\left(k \mathbf{e}-\mathbf{e}_{s}-(\boldsymbol{\pi}-\boldsymbol{\tau})\right)$ is a perfect point for the permuted residual series $R_{\tau}^{c}(z)$.

Let us assume that $B_{\delta}\left(R_{\tau}^{c} ; z\right)$ is the canonical $\delta$-reduced basis matrix for the perfect point $\delta$ in the vector Padé-Hermite table for $R_{\tau}^{c}(z)$. The $\delta$-column degrees of the columns of $B_{\delta}\left(R_{\tau}^{c} ; z\right)$ for a perfect point $\delta$ are given as $\mathbf{e}_{s}+\operatorname{proj}(\delta)-\delta$ which is equal to $\mathbf{e}_{s}+\left(\boldsymbol{\pi}^{\prime}-\boldsymbol{\tau}-k \mathbf{e}\right)$ as we shall now show.

There are two possibilities for each component of $\pi$ and $\tau$.

1. $\tau_{i}=\pi_{i} \geq 0$. Hence

$$
\begin{aligned}
(\operatorname{proj}(\boldsymbol{\delta})-\boldsymbol{\delta})_{i} & =\operatorname{proj}\left(k \mathbf{e}-\mathbf{e}_{s}\right)_{i}-\left(k \mathbf{e}-\mathbf{e}_{s}\right)_{i} \\
& =0 \\
& =\operatorname{proj}(\tau+k \mathbf{e})_{i}-(\tau+k \mathbf{e})_{i} \\
& =\pi_{i}^{\prime}-(\tau+k \mathbf{e})_{i} ;
\end{aligned}
$$

2. $\tau_{i}<\pi_{i}=0(s+1 \leq i \leq m)$. Therefore,

$$
\begin{aligned}
(\operatorname{proj}(\boldsymbol{\delta})-\boldsymbol{\delta})_{i} & =\operatorname{proj}(\boldsymbol{\tau}+k \mathbf{e})_{i}-(\boldsymbol{\tau}+k \mathbf{e})_{i} \\
& =\pi_{i}^{\prime}-(\tau+k \mathbf{e})_{i} .
\end{aligned}
$$

Hence, we have shown that

$$
\operatorname{proj}(\delta)-\delta=\pi^{\prime}-(\tau+k \mathbf{e})
$$

In the sequel, we prove that if $B_{\delta}\left(R_{\tau}^{c} ; z\right)$ is connected to the perfect point $\delta$ for $R_{\tau}^{c}(z)$, the polynomial matrix $B_{\tau}(F ; z) B_{\delta}\left(R_{\tau}^{c} ; z\right)$ is a $(\tau+k \mathbf{e})$-reduced basis matrix connected to the perfect point $(\tau+k \mathbf{e})$ for $F(z)$. First, we show that the correct number of interpolation conditions is satisfied, i.e., we have to show that

$$
\left\|\boldsymbol{\pi}^{\prime}\right\|+s=(\|\boldsymbol{\pi}\|+s)+(\|\operatorname{proj}(\delta)\|+s) .
$$

Using (10), we get

$$
\begin{aligned}
(\|\boldsymbol{\pi}\|+s)+(\|\operatorname{proj}(\delta)\|+s) & =(\|\boldsymbol{\pi}\|+s)+\left(\|\boldsymbol{\delta}\|+\left\|\boldsymbol{\pi}^{\prime}\right\|-\|\boldsymbol{\tau}+k \mathbf{e}\|+s\right) \\
& =(\|\boldsymbol{\pi}\|+s)+\left[\left(\left\|-\mathbf{e}_{s}\right\|-\|\boldsymbol{\pi}\|+\|\boldsymbol{\tau}+k \mathbf{e}\|\right)\right. \\
& \left.\left.+\left\|\boldsymbol{\pi}^{\prime}\right\|-\|\boldsymbol{\tau}+k \mathbf{e}\|+s\right] \text { (def. of } \delta\right) \\
& =\left\|\boldsymbol{\pi}^{\prime}\right\|+s .
\end{aligned}
$$

In the remaining part of the proof, we show that $B_{\tau}(F ; z) B_{\delta}\left(R_{\tau}^{c} ; z\right)$ is $(\tau+k \mathbf{e})$-reduced with $(\tau+k \mathbf{e})$-column degrees $\mathbf{e}_{s}+\boldsymbol{\pi}^{\prime}-(\tau+k \mathbf{e})$. Because $B_{\tau}(F ; z)$ is $\tau$-reduced with $\tau$-column degrees $\mathbf{e}_{s}+\pi-\tau$, and $B_{\delta}\left(R_{\tau}^{c} ; z\right)$ is $\delta$-reduced with $\delta$-column degrees $\mathbf{e}_{s}+\pi^{\prime}-(\tau+k \mathbf{e})$, we can write

$$
\begin{aligned}
B_{\tau}(F ; z) B_{\delta}\left(R_{\tau}^{c} ; z\right) & =\left(z^{\tau} B_{\tau, h}(F ; z) z^{\mathbf{e}_{s}+\pi-\tau}\right)\left(z^{k \mathbf{e}-\mathbf{e}_{s}-\pi+\tau} B_{\delta, h}\left(R_{\tau}^{c} ; z\right) z^{\mathbf{e}_{s}+\pi^{\prime}-\tau-k \mathbf{e}}\right) \\
& =z^{\tau+k \mathbf{e}} B_{\tau, h}(F ; z) B_{\delta, h}\left(R_{\tau}^{c} ; z\right) z^{\mathbf{e}_{s}+\pi^{\prime}-\tau-k \mathbf{e}}
\end{aligned}
$$


with $\operatorname{deg} \operatorname{det}\left(B_{\tau, h}(F ; z) B_{\delta, h}\left(R_{\tau}^{c} ; z\right)\right)=0$ and $B_{\tau, h}(F ; z) B_{\delta, h}\left(R_{\tau}^{c} ; z\right)=O_{-}\left(z^{0}\right)$. Hence, $B_{\tau}(F ; z) B_{\delta}\left(R_{\tau}^{c} ; z\right)$ is $(\tau+k \mathbf{e})$-reduced with $(\tau+k \mathbf{e})$-column degrees $\mathbf{e}_{s}+\pi^{\prime}-(\tau+k \mathbf{e})$. It is easy to see that if $B_{\tau}(F ; z)$ and $B_{\delta}\left(R_{\tau}^{c} ; z\right)$ are both the canonical basis matrices in the sense of Theorem 19 , their product is also the canonical basis matrix. This proves the "if" part of the theorem.

The "only if" part is proved similarly.

Marc Van Barel, Adhemar Bultheel

Department of Computer Science

Katholieke Universiteit Leuven

Celestijnenlaan 200A

B-3001 Heverlee

Belgium 